\title{
Asymptotically optimal Berry-Esseen-type bounds for distributions with an absolutely continuous part
}

\author{
Michael V. Boutsikas \\ Department of Statistics and Insurance Science, University of Piraeus, Greece
}

\begin{abstract}
Recursive and closed form upper bounds are offered for the Kolmogorov and the total variation distance between the standard normal distribution and the distribution of a standardized sum of $n$ independent and identically distributed random variables. The approximation error in the CLT obtained from these new bounds vanishes at a rate $O\left(n^{-k / 2+1}\right)$, provided that the common distribution of the summands possesses an absolutely continuous part, and shares the same $k-1(k \geq 3)$ first moments with the standard normal distribution. Moreover, for the first time, these new uniform Berry-Esseen-type bounds are asymptotically optimal, that is, the ratio of the true distance to the respective bound converges to unity for a large class of distributions of the summands. Thus, apart from the correct rate, the proposed error estimates incorporate an optimal asymptotic constant (factor). Finally, three illustrative examples are presented along with numerical comparisons revealing that the new bounds are sharp enough even to be used in practical statistical applications.
\end{abstract}

Abbreviated Title: Optimal Berry-Esseen-type bounds.

Key words and phrases: Central limit theorem, Berry-Esseen theorem, Edgeworth expansions to the CLT, Rate of convergence, Kolmogorov and total variation distance, Zolotarev's ideal metric.

AMS 2000 subject classification: Primary: 60F05

Secondary: 60G50; 60E15; 62E17; 62G20

\section{Introduction}

One of the most remarkable results in the field of probability and statistics is the Central Limit Theorem (CLT). Important role for the deeper investigation, as well as for the practical use of this celebrated asymptotic result, plays the establishment of the associated rates of convergence. These are usually expressed through asymptotic (Edgeworth) expansions or explicit (Berry-Esseen-type) bounds. The former provide better asymptotic accuracy for the approximation error, but they incorporate terms in the expansion which do not possess an explicit closed form (for example, are expressed via $o\left(n^{-p}\right)$ ). Therefore, Edgeworth expansions are practically useless for fixed values of the number $n$ of summands. On the other hand, Berry-Esseen-type bounds offer explicit approximation errors for every value of $n$, however usually these are very crude. The purpose of the present paper is to unify the advantages of the two aforementioned approaches. That is, to develop Berry-Esseen-type bounds (both for uniform and local error estimates) which, for the first time, exhibit the same asymptotic accuracy as the optimal Edgeworth expansions, under certain weak assumptions. 
To be more specific, let $X, X_{1}, X_{2}, \ldots$ be independent and identically distributed (i.i.d.) random variables (r.v.'s) with $\mathbb{E} X=0, \mathbb{E} X^{2}=1$ and $\mathbb{E}|X|^{3}<\infty$. Denote by $F_{n}$, the cumulative distribution function (c.d.f.) of the standardized sum $n^{-1 / 2} \sum_{i=1}^{n} X_{i}$ and by $\Phi$ the c.d.f. of the standard normal law. The classical uniform Berry-Esseen theorem (see Berry (1941) and Esseen (1942)) states that

$$
\sup _{x}\left|F_{n}(x)-\Phi(x)\right| \leq C \frac{\mathbb{E}|X|^{3}}{\sqrt{n}},
$$

for some constant $C>0$. The smallest known value of $C$ fulfilling the above is 0.7056 (Shevtsova (2007)). If we further assume that $X$ follows a non-lattice distribution then the one-term Edgeworth expansion of the c.d.f. $F_{n}$ of $n^{-1 / 2} \sum_{i=1}^{n} X_{i}$ is (for example, see Petrov (1995)),

$$
F_{n}(x)=\Phi(x)+\frac{\mathbb{E} X^{3}\left(1-x^{2}\right) e^{-x^{2} / 2}}{3 ! \sqrt{2 \pi n}}+o\left(\frac{1}{\sqrt{n}}\right),
$$

uniformly in $x$. Obviously, the above asymptotic estimate is better than the one implied by the Berry-Esseen theorem (note that, $\sup _{x}\left|1-x^{2}\right| e^{-x^{2} / 2}=1$ ). However, contrary to (1), the asymptotic expansion (2) is useless in daily statistical practices where we are interested in estimates of the difference $F_{n}(x)-\Phi(x)$ for a fixed value of $n$. For example, relation (2) cannot be exploited when we want to determine the number $n$ of summands needed for achieving a prescribed degree of approximation accuracy. A finer account of the comparison between Berry-Esseen-type results and Edgeworth expansions can be found in Adell and Lekuona (2008) (see also Seoh and Hallin (1997)).

Traditionally, the standard way for establishing closed form error estimates in the CLT is through Fourier analytic methods (for example, see Petrov(1995)). However, Fourier techniques can be considered indirect and rather intricate and, as a result, several other alternative methods were developed in the last decades. The most important among them are: (i) the approach based on the elegant theory of probability metrics developed by V.M. Zolotarev and his colleagues and students (for example see Rachev (1991) and the references therein) and (ii) the celebrated Stein's method, originally developed by Stein (1970) and extended by many authors in various distribution approximation directions, including the case of dependent summands (for example see Barbour and Hall (1984), Chen and Shao (2001), Barbour and Chen (2005) and the references therein). We also mention the approach through covariance kernels or $w$-functions (for example see Cacoullos, Papadatos and Papathanasiou (1997)) which can be considered that is related to Stein's method. But, despite the great interest and the vast literature concerning Berry-Esseen theorems, to the best of our knowledge, until now there do not seem to exist in the literature asymptotically optimal Berry-Esseen-type bounds. Note that, an upper bound for some distance can be considered asymptotically optimal when the ratio of the bound to the true distance converges to unity as $n \rightarrow \infty$. That is, the bound not only possesses the correct rate of convergence but, in addition, it incorporates an asymptotically optimal constant (factor). An attempt towards this goal was recently made by Adell and Lekuona (2008) who presented "near optimal" Berry-Esseen-type bounds for the uniform distance between $F_{n}$ and $\Phi$. Their bounds were obtained by substituting Esseen's smoothing inequality by an inequality involving the second modulus of continuity.

In the present paper, motivated by the comparison between Edgeworth expansions and BerryEsseen bounds, we develop recursive and closed form upper bounds for the uniform (or Kolmogorov) and the total variation distance between the distribution of $n^{-1 / 2} \sum_{i=1}^{n} X_{i}$ and $\Phi$. These bounds 
are proven to be asymptotically optimal for a large class of distributions of the summands. More specifically, suppose that $\mathbb{E} X^{i}=\mathbb{E} Z^{i}, i=1,2, \ldots, k-1$ for some integer $k \geq 3$, where $Z$ is a r.v. following a standard normal law. Henceforth, the standard normal law will be denoted by $\mathcal{N}$ and the law of a r.v. $X$ will be denoted by $\mathcal{L} X$. If $\mathcal{L} X$ has an absolutely continuous part, we derive an explicit bound $\mathbf{R}_{k, n}$ for the uniform distance (see Theorem 10 and Corollary 13 below) such that

$$
\sup _{x}\left|F_{n}(x)-\Phi(x)\right| \leq \mathbf{R}_{k, n} \sim \frac{\left\|\varphi^{(k-1)}\right\|_{\infty}}{n^{k / 2-1}} \zeta_{k}(\mathcal{L} X, \mathcal{N})=\frac{\left\|\varphi^{(k-1)}\right\|_{\infty}}{n^{k / 2-1}} \frac{\left|\mathbb{E} X^{k}-\mathbb{E} Z^{k}\right|}{k !}
$$

The quantity $\left\|\varphi^{(i)}\right\|_{\infty}$ denotes the $L_{\infty}$-norm of the $i$ th-order derivative of the probability density function (p.d.f.) $\varphi$ of $\mathcal{N}$, and $\zeta_{k}$ denotes the Zolotarev's ideal metric of order $k$. As usual, the notation $a_{n} \sim b_{n}$ implies that $a_{n} / b_{n} \rightarrow 1$ (or whenever $a_{n}=0$ then $b_{n}=0$ and vice versa). The last equality in (3) is valid if, in addition, $\mathcal{L} X$ and $\mathcal{N}$ are $k$-convex ordered (see Boutsikas and Vaggelatou (2002)). This stochastic ordering can usually be easily checked, and seems to be satisfied by many known distributions (see Remark 3 below). When $k=3$ (that is, $\mathbb{E} X=0, \mathbb{E} X^{2}=1$ ), a condition that is always valid after standardization of the summands, relation (3) reduces to

$$
\sup _{x}\left|F_{n}(x)-\Phi(x)\right| \leq \mathbf{R}_{3, n} \sim \frac{\zeta_{3}(\mathcal{L} X, \mathcal{N})}{\sqrt{2 \pi n}}=\frac{\left|\mathbb{E}^{3}\right|}{3 ! \sqrt{2 \pi n}} .
$$

The last equality is again valid when $\mathcal{L} X$ and $\mathcal{N}$ are 3 -convex ordered (for example, when $\mathcal{L} X$ is the gamma distribution; see example (a) in Section 5). Remarkably, the above asymptotic form of the bound $\mathbf{R}_{3, n}$ is consistent to that implied by the Edgeworth expansions (see (2) above), and $\mathbf{R}_{3, n}$, or more generally $\mathbf{R}_{k, n}$, is indeed proven to be asymptotically optimal (see Corollary 13 below).

It is also remarkable that, when $\mathcal{L} X$ share higher order moments with $\mathcal{N}$ (that is, $k>3$ ), the vanishing rate of $\mathbf{R}_{k, n}$ is amplified. If, for example, $\mathcal{L} X$ is symmetric, then $\mathbb{E} X^{3}=0$ and therefore, after standardization of $X$, relation (3) is valid for some $k$ at least equal to 4 (see example (b) in Section 5 regarding the uniform distribution).

Analogous results are proven for the total variation distance $\mathbf{d}_{n}$ between the law of the standardized sum of $n X_{i}$ 's and $\mathcal{N}$. In particular, according to Theorem 4 below, when for some integer $k \geq 3$ we have $\mathbb{E} X^{i}=\mathbb{E} Z^{i}, i=1,2, \ldots, k-1$ and $\mathcal{L} X$ has an absolutely continuous part, then $\mathbf{d}_{n}$ is bounded above by an explicit bound $\mathbf{D}_{k, n}$, such that

$$
\mathbf{d}_{n} \leq \mathbf{D}_{k, n} \sim \frac{\left\|\varphi^{(k)}\right\|}{2 n^{k / 2-1}} \boldsymbol{\zeta}_{k}(\mathcal{L} X, \mathcal{N})=\frac{\left\|\varphi^{(k)}\right\|}{2 n^{k / 2-1}} \frac{\left|\mathbb{E} X^{k}-\mathbb{E} Z^{k}\right|}{k !},
$$

where now $\|\cdot\|$ denotes the $L_{1}$-norm. Again, the last equality is valid if, in addition, $\mathcal{L} X$ and $\mathcal{N}$ are $k$-convex ordered. In the weakest case, when $k=3$, the above relation reduces to

$$
\mathbf{d}_{n} \leq \mathbf{D}_{3, n} \sim \frac{1+4 e^{-3 / 2}}{\sqrt{2 \pi n}} \zeta_{3}(\mathcal{L} X, \mathcal{N})=\frac{1+4 e^{-3 / 2}}{\sqrt{2 \pi n}} \frac{\left|\mathbb{E} X^{3}\right|}{3 !}
$$

and the bound $\mathbf{D}_{3, n}$ is again proven to be asymptotically optimal (see Corollary 7 ) when $\mathcal{L} X$ and $\mathcal{N}$ are 3 -convex ordered.

For the proof of our main results we proceed to a refinement and an adaptation to the continuous case of the method employed by Boutsikas and Vaggelatou (2009) for compound Poisson approximations. This approach is based on the theory of probability metrics and especially on Lindeberg's decomposition and certain smoothing inequalities satisfied by Zolotarev's ideal metric. In 
that way, it can be also be considered that is closely connected to the method used (in more general spaces) by Rachev and Yukich (1989) (see also Rachev (1991) and the references therein). In the present paper we treat the simplest case of i.i.d. summands, but since Boutsikas and Vaggelatou (2009) achieved to apply a discrete version of this method to locally dependent summands, there is hope that our main outcomes could also be extended to that direction in the future.

Finally, it is worth pointing out a shortcoming of our bounds. They are applicable only when the distribution of $X_{i}$ 's is already relatively close to standard Normal. Fortunately, we can always overcome this situation as will be explained in Remark 1 below and practiced in the examples section.

The paper is organized as follows. In the Section 2 we present all necessary notations and auxiliary results needed for the exposition of our main outcomes, concerning properties of probability metrics, derivative norms of the standard normal density, Hermite polynomials and $s$-convex orders. In the main results section (Section 3) we offer recursive and closed form upper bounds for the Kolmogorov and the total variation distance of interest, along with some results guaranteeing asymptotic optimality. The proofs of the main results, together with all necessary lemmas, are postponed to the next Section 4. The applicability and the performance of our main results is investigated by way of three illustrative applications in Section 5 .

\section{References}

[1] Adell J.A. and Lekuona A. (2008) Shortening the distance between Edgeworth and BerryEsseen in the classical case. Journal of Statistical Planning and Inference, 138, 1167 - 1178.

[2] A.D. Barbour and L.H.Y.Chen (2005) An introduction to Stein's method, Lecture Notes Series, Institute for Mathematical Sciences, National University of Singapore, Singapore university press and world scientific publishing, Singapore.

[3] Barbour A.D. and Hall P. (1984) Stein's method and the Berry-Esseen theorem. Australian J. Statist. 26, 8-15.

[4] Berry, A.C. (1941) The accuracy of Gaussian approximation to the sum of independent variates. Trans. American Math. Soc. 4, 122-136.

[5] Boutsikas, M.V. and Vaggelatou, E. (2002). On the distance between convex-ordered random variables. Adv. Appl. Probab. 34, 349-374.

[6] Boutsikas, M.V. and Vaggelatou, E. (2009) A new method for obtaining sharp compound Poisson approximation error estimates for sums of locally dependent random variables. Bernoulli (to appear)

[7] Cacoullos T., Papadatos N. and Papathanasiou V. (1997) Variance Inequalities for covariance kernels and applications to Central Limit Theorems. Theory Probab. Appl. 42, 149-155.

[8] Chen L.H.Y. and Shao Q.M. (2001) A non-uniform Berry-Esseen bound via Stein's method. Probab. Theory Related Fields, 120, 236-254.

[9] Denuit M., Lefèvre C. and Shaked M. (1998) The $s$-convex orders among real random variables, with applications. Mathematical Inequalities $\&$ Applications 1, 585-613. 
[10] Esseen, C.G. (1942). On the Liapounov limit of error in the theory of probability. Ark. Mat. Astr. Fy. 28A, 1-19.

[11] Petrov, V.V. (1995). Limit Theorems of Probability Theory. Clarendon Press, Oxford.

[12] Prokhorov Yu V. (1952) On a local limit theorem for densities. Dokl. Akad. Nauk SSSR, 83, 797-800.

[13] Rachev S.T. (1991) Probability Metrics and the Stability of Stochastic Models. Wiley, Chichester, New York.

[14] Rachev S.T. and Ruschendorf L. (1990) Approximation of sums by compound Poisson distributions with respect to stop-loss distances. Advances in Applied Probability 22, 350-374.

[15] Rachev S.T. and Yukich J.E. (1989) Rates for the CLT via new ideal metrics. Ann. Prob., 17, 775-788.

[16] Senatov V.V. (1980) Uniform estimates of the rate of convergence in the multi-dimensional CLT. Theory Prob. Appl. 25, 745-759.

[17] Seoh M. (2002) On Improving Uspensky-Sherman's Normal Approximation by an EdgeworthExpansion Approximation. Bull. Belg. Math. Soc. 9, 65-72.

[18] Seoh, M. and Hallin, M. (1997) When does Edgeworth beat Berry and Esseen? Numerical evaluations of Edgeworth expansions. J. Statist. Plann. Inference 63, 19-38.

[19] Shaked, M. and Shanthikumar, J.G. (1994) Stochastic orders and their applications. Academic Press, New York.

[20] Sherman, R. (1971). Error of the normal approximation to the sum of $N$ random variables. Biometrika, 58, 396-398.

[21] Shevtsova I.G. (2007) Sharpening of the upper bound of the absolute constant in the BerryEsseen inequality. Theory Probab. Appl., 51, 549-553.

[22] Sirazhdinov S.Kh. and Mamatov M. (1962) On convergence in the mean for densities. Theory Probab. Appl., 7, 424-429.

[23] Stein, C. (1970) A bound for the error in the normal approximation to the distribution of a sum of dependent random variables, Proc. Sixth Berkeley Symp. Math. Stat. Prob., 2, 583-602, Univ. California Press. Berkeley, Calif.

[24] Uspensky, J.V. (1937) Introduction to Mathematical Probability. McGraw-Hill, New York.

[25] Zolotarev V.M. (1983) Probability metrics. Theory Prob. Appl., 28, 278-302.

\section{Michael V. Boutsikas}

Postal address: Department of Statistics and Insurance Science, University of Piraeus, Karaoli and Dimitriou str. 80, Piraeus 18534, Greece email address: mbouts@unipi.gr 